\title{
Diagnosis and Treatment of Pulmonary Embolism in Challenging Populations
}

\author{
Sebastian Werth ${ }^{1}$ Jan Beyer-Westendorf ${ }^{2,3}$ \\ ${ }^{1}$ Center for Vascular Diseases, Division of Angiology, Department of \\ Medicine III, University Hospital “Carl Gustav Carus” Dresden, \\ Dresden, Germany \\ ${ }^{2}$ Thrombosis Research Unit, Division of Haematology, Department of \\ Medicine I, University Hospital "Carl Gustav Carus" Dresden, \\ Dresden, Germany \\ ${ }^{3}$ Kings Thrombosis Service, Department of Haematology, \\ Kings College London, London, United Kingdom \\ Hämostaseologie 2018;38:87-97.
}

\author{
Address for correspondence Jan Beyer-Westendorf, MD, Thrombosis \\ Research Unit, Division of Haematology, Department of Medicine I, \\ University Hospital “Carl Gustav Carus," Technical University Dresden, \\ Fetscherstrasse 74, 01307 Dresden, Germany \\ (e-mail: jan.beyer@uniklinikum-dresden.de).
}

\author{
Abstract \\ Keywords \\ - pulmonary embolism \\ - pregnancy \\ - cancer \\ - chronic kidney \\ disease
}

\section{Zusammenfassung}

\section{Schlüsselwörter}

- Lungenembolie

- Schwangerschaft

- Krebserkrankung

- chronische Nierenerkrankung
Although the diagnostic and therapeutic approach to pulmonary embolism (PE) has been considerably improved and standardized in recent years, special populations such as pregnant patients and those with impaired renal function or cancer still represent a clinical challenge and a detailed knowledge about the available diagnostic and therapeutic alternatives is mandatory to provide best evidence-based care for these difficult-to-treat patients. Although this review aimed to summarize the most important aspects in this field, the reader is referred to the original studies cited here and dedicated guideline and guidance documents for more detailed information.

Obwohl in den letzten Jahren Diagnostik und Therapie der Lungenembolie deutlich verbessert und standardisiert wurden, stellen Patienten in der Schwangerschaft, mit eingeschränkter Nierenfunktion oder einer Krebserkrankung immer noch eine klinische Herausforderung dar, und ein detailliertes Wissen um verfügbare diagnostische und therapeutische Alternativen ist Voraussetzung, um eine gute evidenz-basierte Versorgung dieser schwer zu behandelnden Patienten zu gewährleisten. Wenngleich diese Übersichtsarbeit die wichtigsten Aspekte hierzu zusammenzufasst, wird der Leser für weitergehende Informationen auf Originalarbeiten sowie Leitlinien zu diesem Thema verwiesen.

\section{Introduction}

Although pulmonary embolism (PE), as a manifestation of venous thromboembolism (VTE), is a common condition and evidence for diagnostic workup and treatment is extensive and summarized in dedicated guidelines, ${ }^{1,2}$ some common clinical scenarios are especially challenging, because standard diagnostic approaches to suspected PE, i.e. pre-test probability, followed by D-dimer testing and/or imaging, may not be feasible (for instance, in pregnancy or cancer patients); because some PE patients may be at higher risk for thromboembolic or bleeding complications (such as cancer or patients witch chronic kidney disease [CKD]) or because standard anticoagulants may be contraindicated (such as vitamin K antagonists [VKAs] or direct, non-vitamin Kaffecting oral anticoagulants [NOACs] in pregnant PE patients). The following review will discuss the most common challenges of PE diagnosis and treatment in pregnancy, active cancer or CKD. For a more detailed guidance, the reader is referred to dedicated guideline and guidance documents. received

August 1, 2017

accepted after revision

January 17, 2018
Copyright () 2018 Schattauer

DOI https://doi.org/

10.1055/s-0038-1641586.

ISSN 0720-9355. 


\section{Diagnosis and Treatment of PE in Pregnancy}

\section{Special VTE Epidemiology in Pregnancy}

Venous thromboembolism still is one of the leading causes of maternal death in the western world. ${ }^{3}$ With an incidence of $1.2 / 1,000$ pregnancies, VTE is a common complication of pregnancy, which translates into a fivefold risk compared with non-pregnant women. ${ }^{4}$ A relevant proportion of VTE in pregnancy manifests as $\mathrm{PE}$, which will affect both maternal and foetal health and the highest risk for thromboembolic complications is observed between the 38th gestational week and 6 weeks post-delivery, when the risk increase is up to 20 -fold of that of non-pregnant women. ${ }^{5}$ Of note, in pregnancies following in vitro fertilization (IVF), the risk of VTE is even higher: in a large cross-sectional study from Sweden, IVF was associated with an overall VTE risk of 4.2/1,000 women (vs. 2.5/1,000 in women with natural pregnancies; hazard ratio [HR] 1.77, 95\% confidence interval [CI] 1.41-2.23) (Henriksson P, BMJ 2013; 346:e8632). Although this risk increase was observed during the whole pregnancy, it was particularly increased during the first trimester (HR, 4.22), during which VTE much more often manifested as PE (0.3/1,000 after IVF vs. 0.04/1,000; HR, 6.97, 95\% CI, 2.21-21.96).

Although diagnosis and treatment of PE have seen important improvements over the last two decades, management of suspected or confirmed PE in pregnant patients is still challenging for various reasons, most of which are highlighted below.

\section{Challenge 1: Overlap of Symptoms of Pregnancy and VTE and Failure of Diagnostic Algorithms}

One of the major challenges in pregnancy relates to the physiological cardiopulmonary changes and their corresponding symptoms. As pregnancy progresses, lower extremity pain and oedema, exertional dyspnoea, tachycardia and palpitations are common. Consequently, established pre-test probability scores such as the Wells score ${ }^{6,7}$ for suspected deep vein thrombosis (DVT) or the Geneva score for suspected $\mathrm{PE}^{8}$ are of limited value in assessing the likelihood of VTE. Furthermore, D-dimer physiologically increase during pregnancy ${ }^{9,10}$ so that established VTE diagnostic algorithms ('negative D-dimer can safely rule out VTE in patients with low pre-test probability scores') are of little help in pregnant patients with suspected VTE. Although this algorithm statement per se is also correct for pregnant patients with suspected PE, the clinical feasibility is impaired by the fact that negative D-dimer levels are rare in pregnant women. Pregnancy-specific algorithms or pregnancy-adjusted D-dimer cut-offs have been suggested ${ }^{11}$ but, so far, they need further validation before their use can be recommended. Consequently, the approach to suspected PE in pregnant patients requires dedicated clinical experience.

\section{Challenge 2: Potential Harm from Radiation or Contrast Media in Diagnostic Procedures}

Due to the potential maternal and foetal harm, every suspicion of VTE needs immediate, safe and effective confirmation or exclusion. Because of the limitations of pre-test probability scores and D-dimer testing, imaging procedures are the main- stay of the diagnostic process. However, the accuracy of venous ultrasound is again limited, since only a relatively small proportion of VTE in pregnant patients manifest as infra-inguinal DVT which can be easily detected by compression ultrasound. ${ }^{12,13}$ Iliac DVT is relatively common in pregnant women but ultrasound examination of iliac veins is challenging, also because of the pregnancy-related anatomical changes which may lead to venous compression. ${ }^{13}$ Diagnostic alternatives include computed tomography (CT) or magnetic resonance (MR) venography for iliac vein imaging or CT pulmonary angiography or lung scintigraphy (ventilation-perfusion [VQ] scan) for pulmonary arteries. Although well established, these imaging procedures carry specific risks. CT scanning requires the use of contrast media with the risk of anaphylactic reactions, hyperthyroidism or renal injury. Furthermore, the radiation exposure to the pelvic region (for iliac vein imaging) or to the chest (for suspected PE) increases the risk for radiation embryopathy or breast cancer. ${ }^{14,15} \mathrm{~A}$ lower maternal radiation exposure can be expected from VQ scanning but the foetal radiation exposure is higher, since radioactive tracers will pass the placenta. MR imaging does not carry the radiation risk but the most commonly used contrast media such as gadolinium cross the placenta and embryotoxicity cannot completely be ruled out. ${ }^{16}$ Finally, MR imaging of the pulmonary vasculature has not been sufficiently validated in pregnant women with suspected PE.

As a consequence of all these challenges, the diagnostic approach to PE in pregnancy needs both standardization and an individualized, tailored approach based on clinical experience with this clinical scenario. Therefore, such patients should be referred to a specialist care setting because, in a pregnant patient with suspected PE, it is most important to definitively rule out (or confirm PE), since over- or under-treatment with anticoagulants may have deleterious implications for the mother and child. A specialized centre will also have CT protocols established that are dedicated to reduce CT-related radiation dosages for mother and foetus so that the fear of CTrelated radiation exposure should no longer exclude a pregnant women from adequate PE diagnosis via CT.

\section{Recommended Diagnostic Strategy for Suspected PE in Pregnancy}

If PE is suspected in pregnant women, D-dimer testing (may still be negative in early stages of pregnancy to rule out PE), compression ultrasound (may establish the presence of DVT and, therefore, the indication for anticoagulant therapy without the need for lung scanning) and echocardiography (may be used to detect signs of otherwise unexplained right heart strain or pulmonary hypertension) should be applied first, before the exposure to radiation or contrast media is considered. If these tests remain inconclusive (which is often the case in suspected pregnancy related PE!) and the clinical suspicion justifies further evaluation, radiological imaging procedures should not be withheld. First, a standard chest X-ray should rule out non-PE pathologies such as pleural effusion, pneumonia or pneumothorax. After a normal X-ray result, lung perfusion scintigraphy is sufficient to safely rule out PE, since cardiopulmonary co-morbidities requiring classic VQ scan 
are very rare in younger women and, by elimination the ventilation part, radiation exposure as well as examination times can be reduced. If lung perfusion scintigraphy is not readily available or the chest X-ray shows pathological findings, CT pulmonary angiography should be used as the next step. If dedicated test protocols are applied, both lung perfusion scintigraphy and CT pulmonary angiography carry a foetal radiation exposure considerably below $50 \mathrm{mSv}$, which is the commonly described threshold for radiation-related congenital abnormalities and miscarriages. ${ }^{15}$ Of note, a higher cardiac output needs to be considered in CT protocols in late pregnancy to achieve adequate contrast in the pulmonary vasculature.

-Fig. 1 summarizes a feasible clinical pathway for pregnant women with suspected PE. Of note, this pathway is based on expert recommendation and, so far, has not been fully validated.

Recommended Therapy for Pregnancy-Associated PE Although inconvenient and costly, anticoagulant therapy in pregnancy-associated VTE is usually performed with lowmolecular-weight heparin (LMWH) or fondaparinux, ${ }^{17}$ because these drugs have been used in pregnancy for nearly two decades with low rates of severe maternal or foetal complications, ${ }^{18}$ whereas all oral anticoagulants have serious limitations, which mainly relate to the placenta-crossing of VKAs and direct acting, NOACs. In addition to placental and foetal haemorrhage, coumarin embryopathy is a reported complication especially with VKA exposure between 6 and 12 weeks after last menstrual period ${ }^{19}$ and reported abnormalities include mid-face hypoplasia, ocular malformations and skeletal abnormalities. ${ }^{20,21}$ Older studies suggested rates up to $30 \%^{22}$ but more recent data estimate a $7 \%$ risk for coumarin embryopathy.

NOACs are rapidly becoming the standard of care in most VTE patients, because, compared with VKA, they offer a favourable efficacy/safety profile and better convenience. However, NOACs are small molecules that cross the placen$\mathrm{ta}^{23-25}$ and the clinical risk of NOAC embryopathy is currently unknown. NOAC exposure in animals indicated a risk of reproductive toxicity at therapeutic to toxic dosages, which manifested as a decrease in implantations, increased implantation loss, malformations, altered ossification and haemorrhagic complications. ${ }^{23,24,26}$

The available data on NOAC exposure in pregnant patients are very limited ${ }^{23,24,26-28}$ and, although the risk of embryotoxicity seems to be rather low, ${ }^{29}$ the use of NOAC during pregnancy or breastfeeding cannot be recommended. ${ }^{30}$

Because of these serious limitations of oral anticoagulants, LMWH are the treatment of choice for VTE during pregnancy. ${ }^{18}$ Similar to the treatment of non-pregnant patients, LMWH dosing in pregnancy is adjusted to body weight. Measurement of anti-factor Xa plasma levels is not generally recommended (also, because target ranges are not well established and pharmacokinetics of LMWH change as pregnancy progresses, due to a change in distribution volume and kidney function) but may help to guide LMWH dosing in difficult situations such as extremely high or low body weights, hereditary antithrombin deficiency or renal impairment.

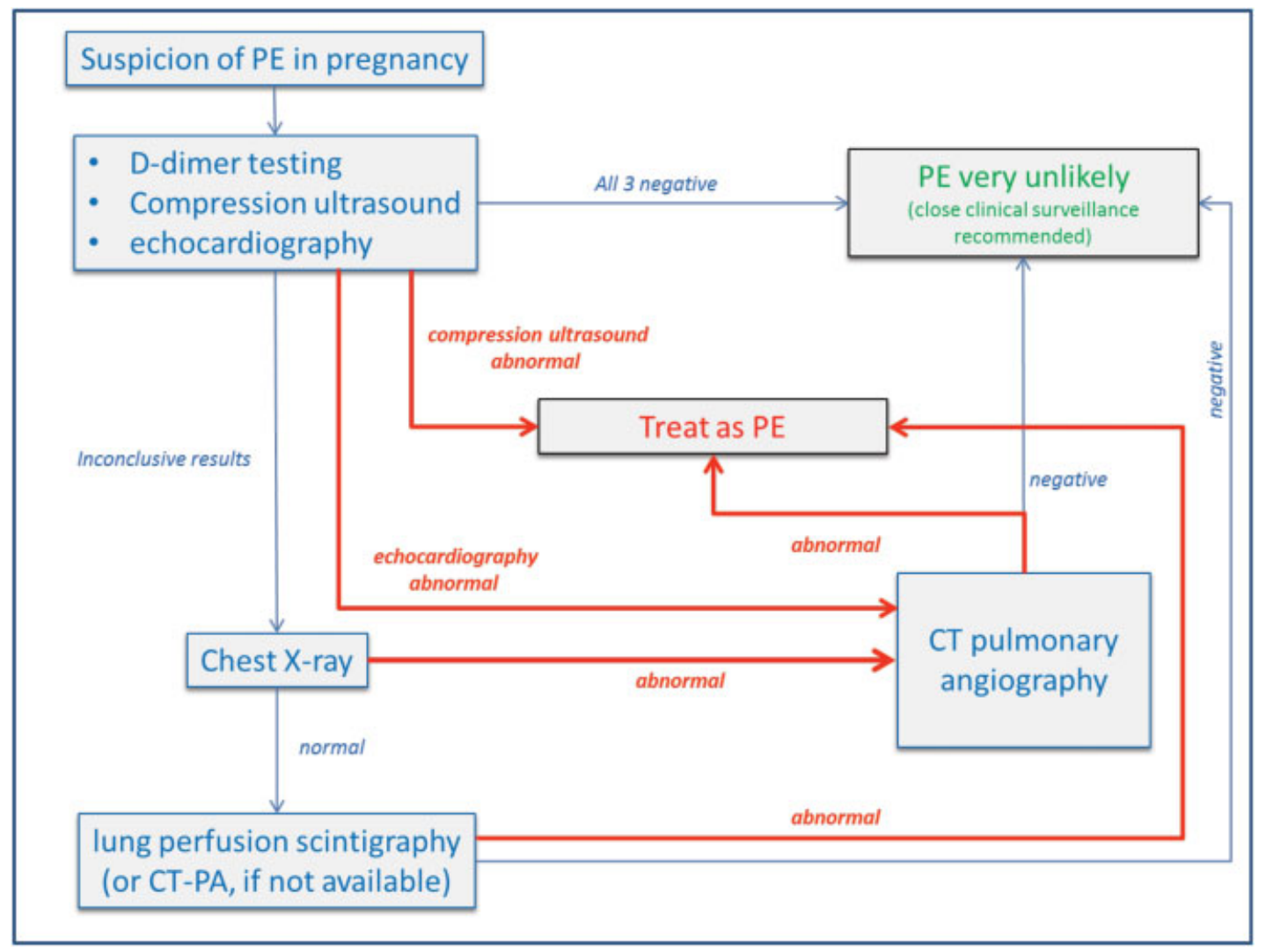

Fig. 1 Proposed diagnostic algorithm for suspected pulmonary embolism (PE) in pregnancy. 
To treat pregnancy-associated VTE, LMWH should be administered in therapeutic dosages (175-200 aXa units/kg/day) for at least 3 months. ${ }^{18}$ In case of VTE diagnosis in the early phases of pregnancy, LMWH should be continued until 6 weeks post-delivery but a dose reduction to 100 aXa units/kg/day may be considered after 3 months of therapeutic anticoagulation. If VTE is diagnosed after the 28th gestational week, LMWH should be administered in therapeutic dosages (175-200 aXa units/kg/day) until at least 6 weeks post-delivery or until 3 months of anticoagulation are completed (whichever is longer).

Around delivery, LMWH dosing regimen may be switched to twice daily (lower LMWH peak levels) and interrupted with the onset of labour. If no major bleeding occurs, LMWH can be restarted 6 to 12 hours post-delivery. In high-risk VTE patients, switch to unfractionated heparin infusions and induced delivery or caesarean section may be considered.

\section{Anticoagulation in Cancer Patients with PE}

\section{Epidemiology of Cancer-Associated VTE}

VTE is a significant contributor to morbidity and mortality in cancer patients, ${ }^{31}$ since local vein compression, pro-coagulant effects of cancer and cancer therapies, immobility, medical and surgical interventions and the long-term use of central venous catheters increase the risk of VTE up to sevenfold. ${ }^{32}$ At the same time, active cancer is a strong and independent risk factor for major bleeding, which makes the anticoagulant treatment of cancer-associated VTE (CAT) especially challenging. ${ }^{33,34}$

The incidence of CT is highly variable across different cancer types, with brain, pancreatic and gastric cancer as well as myeloma being associated with very high risk, colon, liver, rectum and lung cancer with high risk and prostate, cervix, uterus, breast and bladder cancer with comparatively low risk, respectively. At the same time, risk of CAT is increasing with advanced stages of cancer and metastatic disease. ${ }^{35,36}$ As with the risk for a primary VTE event, risk of VTE recurrence is dependent on cancer type and stage, ${ }^{37}$ which makes the decision to continue or discontinue anticoagulant therapy challenging.

\section{Diagnosis of PE in Cancer Patients}

Cancer patients with suspected PE should generally undergo objective testing, for which CT is the current standard. In contrast to patients without cancer, the use of clinical prediction models such as the Wells score or the Geneva score is of limited use in cancer patients, because the presence of cancer alone will increase the score. ${ }^{6,8}$ Furthermore, even if these scores indicate a low probability of PE, this result needs to be complemented with low D-dimer values to safely rule out presence of $\mathrm{PE}$, but D-dimer often is elevated in cancer patients. ${ }^{38,39}$ Of note, a negative D-dimer test has the same diagnostic value as in non-cancer patients and there is evidence that raising the D-dimer cut-off level to $700 \mathrm{mg} / \mathrm{L}$ or using age-dependent cut-off levels may help to increase the proportion of cancer patients in whom PE could be ruled out without imaging with an acceptable failure rate. ${ }^{38,39}$ Since the standard diagnostic algorithm of combining pre-test probability scores and D-dimer testing before imaging has limitations, CT scanning is currently the standard approach to establish or rule out PE in most cancer patients. However, many cancer patients also have silent or even bilateral lower extremity DVT at the time of PE suspicion so that a bilateral compression ultrasound of the lower extremities may streamline the diagnosis and treatment decisions in cancer patients, since the presence of DVT and the indication for anticoagulant therapy may reduce the need for CT scanning.

\section{Special Risk for Recurrence and Bleeding}

Cancer patients with PE are at an increased risk for VTE recurrence and major bleeding complications compared with non-cancer PE patients. The rate of recurrence is highest during the first 2 weeks after VTE diagnosis and declines thereafter and a failure to rapidly achieve therapeutic levels of anticoagulation has been shown to be an independent predictor of VTE recurrence. ${ }^{40,41}$ Early mortality after VTE diagnosis is especially high in cancer patients. In the RIETE registry, all-cause 3-month mortality was as high as $26 \%$ for cancer patients and mostly related to active cancer. ${ }^{42}$ However, poor outcome was also shown to be related to the increased bleeding risk during anticoagulation therapy and to the high rate of recurrence of VTE. ${ }^{43}$ Unfortunately, bleeding complications in CAT patients, although a common clinical problem, are difficult to predict. The existing bleeding prediction scores for VTE patients are not well established yet and have included very few or no CAT patients in their derivatization or validation cohorts. The prognostic risk assessment for future bleeding risk in CAT patients thus remains an urgent but unmet clinical need.

However, although cancer patients with PE are at higher risk of thromboembolic and bleeding complications compared with non-cancer PE patients, the clinical approach to the acute phase of PE is independent from cancer status, because a dedicated assessment of PE severity immediately at the time or presentation is crucial to tailor the following treatment decisions in accordance with the short-term risk of right-heart failure. Therefore, PE patients with our without cancer who present in shock should undergo urgent revascularization, although mechanical revascularization may be more frequently considered in cancer PE due to the increased bleeding risk associated with both active cancer and fibrinolytic therapy. Similarly, in low to intermediate risk PE a more conservative approach, consisting of therapeutic anticoagulation and surveillance may be used, irrespective of cancer status. Of note, the risk assessment of PE severity should always be based on validated scores such as Pulmonary Embolism Severity Index (PESI) or simplified PESI (sPESI), ${ }^{44,45}$ all of which include cancer as a prognostic factor. Therefore, the aforementioned risk-adjusted approach to high-, intermediate- or low-risk PE already accounts for the presence or absence of active cancer. On the other hand, the fact that presence of cancer will always result in an increased PESI or sPESI score, CAT patients would generally be excluded from outpatients PE treatment, if the eligibility check for outpatient therapy would solely be based on PESI or sPESI. It seems 
reasonable to allow for outpatient PE treatment also in CAT patients, if no other risk factors are present.

\section{Special Situation 'Incidental PE' in Cancer Staging Examinations}

With the increased availability and sensitivity of CT and MR, scans are increasingly demonstrating asymptomatic DVT or PE ('clinically unsuspected PE' or 'incidental PE') in cancer patients undergoing routine staging examinations. ${ }^{46,47}$ The prevalence of such incidental VTE events has been reported to be in a range of 2 to $6 \% 48,49$ and $~ 40 \%$ of these events are found in lung scans. ${ }^{50}$ Naturally, the prevalence is depending on the type of scan and higher in series that report findings from CT pulmonary angiography. ${ }^{51}$

Although asymptomatic, incidental PE is of clinical relevance. Several studies have demonstrated that patients with incidental VTE seem to be at a similar risk to develop symptomatic VTE or mortality as cancer patients with symptomatic VTE. ${ }^{52,53}$ As a consequence, current guidelines suggest that incidental PE should be treated similarly as symptomatic CAT, ${ }^{1,54}$ which would mean that all cancer patients with incidental PE would receive at least 6 months of anticoagulant therapy or indefinite treatment in case of non-curable cancer. However, no prospective studies have demonstrated a benefit from such a decision and the likelihood of false-positive scan results (especially in segmental and sub-segmental PE) as well as the increased risk of bleeding need to be taken into consideration.

\section{Data for VKA and LMWH in CAT}

Several studies have demonstrated that LMWH achieve superior efficacy over VKA in the treatment of cancer-associated VTE. Several smaller studies as well as the CLOT trial ${ }^{55}$ and, more recently, the Comparison of Acute Treatments in Cancer Hemostasis (CATCH) $\operatorname{trial}^{56}$ cumulatively demonstrated a $40 \%$ relative risk reduction for VTE recurrence ${ }^{57}$ for LMWH compared with warfarin in the treatment of CAT with similar rates for major bleeding. However, at 6 months of therapy absolute rates of VTE recurrence with LMWH were still $7 \%$ for tinzaparin ${ }^{56}$ and $8 \%$ for dalteparin, ${ }^{55}$ which in a cancer population that demonstrated mortality rates between 30 and $40 \%$, which was mainly driven by cancerrelated death. Based on these data, guidelines currently recommend LMWH over VKA in the treatment of cancerassociated VTE but these recommendations have not been updated in the era of NOACs.

\section{Data for NOAC in CAT}

Over the last few years, NOAC have rapidly developed towards a standard therapy in VTE and evidence for NOAC use in CAT is increasing. Post hoc analyses of cancer patients from phase III NOAC trials were summarized in a network meta-analysis, which modelled an indirect comparison against LMWH from the aforementioned LMWH/VKA trials. ${ }^{57}$ This indirect comparison suggested that NOAC could be as effective and safe as LMWH in CAT treatment. Over the last 2 years, real-world studies have provided first evidence for NOAC use in CAT and have demonstrated outcome rates for recurrent VTE and major bleeding that were not higher than the rates in the respective LMWH studies. ${ }^{58-60}$

Recently, two randomized controlled trials (RCTs) comparing NOAC against LMWH have reported outcomes for CAT treatment. The Hokusai Cancer ${ }^{61}$ study was a large, multinational non-inferiority RCT comparing edoxaban versus dalteparin for CAT therapy. A total of 1,050 patients (mean age, 64 years; 98\% active cancer, 53\% metastatic disease) were included and followed-up for 12 months. Rate of recurrent VTE was 7.9 versus $11.3 \%$ ( $\mathrm{HR}, 0.71,95 \% \mathrm{CI}$, $0.48-1.06)$ for edoxaban versus dalteparin. Rate of major bleeding was 6.9 versus $4.0 \%$ ( $\mathrm{HR}, 1.77,95 \% \mathrm{CI}, 1.03-3.04$ ) for edoxaban versus dalteparin.

Another randomized trial in SELECT-D ${ }^{62}$ cancer patients at risk of recurrence of VTE (SELECT-D) evaluated rivaroxaban versus dalteparin in 406 patients (mean age, 67 years; $59 \%$ metastatic disease) over a period of 6 months. Rate of recurrent VTE was 4 versus $11 \%$ for rivaroxaban versus dalteparin. Rate of major bleeding was 5 versus $3 \%$ for edoxaban versus dalteparin. Further RCTs comparing NOAC against LMWH in CAT treatment are currently on-going.

\section{Recommendation for Anticoagulant Treatment in CAT} Although there is increasing evidence for NOAC use in CAT, it should be recognized that, so far, no direct head-to-head comparisons for NOAC versus LMWH are available and that the existing data are likely derived from less sick cancer patients for whom an alternative to daily LMWH injections was sought. Whereas LMWH can be easily dose-adjusted in case of declining body weight, renal impairment or thrombocytopenia, the fixed dosing of NOAC does not allow for such dedicated treatment tailoring in cancer patients. Furthermore, oral anticoagulation may not be feasible in cancer patients affected by nausea or vomiting. Finally, NOAC metabolism is affected by several drugs that are metabolized via CYP3A4 or p-GP, which includes several antibiotic, antimycotic and antiviral drugs, ${ }^{63}$ which are commonly used in cancer patients. Consequently, LMWH offer advantages over oral anticoagulants that go beyond the comparative rates of VTE recurrence or major bleeding and it can be expected that the recommendation to use LMWH as a first line therapy in CAT will probably not change completely. However, based on the currently available evidence, edoxaban and rivaroxaban may be considered as alternatives for patients who are unable or unwilling to continue long-term LMWH therapy. Furthermore, no evidence supports a benefit of LMWH over oral alternatives in the CAT treatment beyond 6 months and a switch to oral anticoagulants may offer such long-term survivors a more convenient and less costly alternative.

\section{Anticoagulation in Patients with Chronic Kidney Disease}

\section{Epidemiology of CKD-PE}

VTE patients with CKD are especially challenging, since they exhibit a higher risk for VTE recurrence and bleeding complications, compared with patients without renal impairment. ${ }^{64}$ 
In a sub-study of the RIETE registry, patients with creatinine clearance $(\mathrm{CrCl})<30 \mathrm{~mL} / \mathrm{min}$ were compared with patients with a $\mathrm{CrCl}$ of 30 and higher and were found to be at higher risk for fatal bleeding $(0.2 \%$ for $\mathrm{CrCl}>30 \mathrm{~mL} / \mathrm{min}$ vs. $1.2 \%$ for $\mathrm{CrCl}<30 \mathrm{~mL} / \mathrm{min}$ ), fatal PE (1.1 vs. 6.6\%, respectively) and overall death (2.6 vs. 16 ) during the 3 -month study period. ${ }^{65}$ As a consequence, anticoagulant treatment options need to provide an optimal net clinical benefit.

\section{Data for VKA and LMWH in CKD-PE}

Most of the available data on VKA anticoagulation in CKD refer to atrial fibrillation patients and data on VTE are scarce. However, the limitations of VKA anticoagulation in advanced CKD stages can be expected to be similar in atrial fibrillation and VTE and mainly relate to high bleeding rates and unstable international normalized ratio (INR) values. Even though, VKA are still widely used in VTE treatment in CKD patients.

In recent years, warfarin was used as the standard of care comparator in all NOAC phase III trials in acute VTE treatment. ${ }^{66-71}$ All these studies consistently reported increase of thromboembolic and bleeding complications for VKA as renal function was declining. Potential explanations for a higher incidence of thromboembolic and bleeding complications may include:

- More unstable INR courses with a higher risk of overdose, which increases the risk of bleeding in CKD. ${ }^{72}$

- Higher age and more complex co-morbidities of CKD patients. $^{73}$

- Harmful side effects of VKA such as accelerated vascular calcification including additional renal decline. ${ }^{74,75}$

However, the evidence for these considerations is weak. In summary, VKA are not providing optimal net clinical benefit for VTE patients with CKD but the underlying pharmacological mechanisms for this established clinical observation are insufficiently understood.

\section{Data for NOAC in CKD-PE}

The pharmacological background of NOAC (fixed daily dosing, strong dose-response correlation, comparatively broad therapeutic window, well-defined interaction with coagulation cascade) suggests that this class of compounds may overcome the limitations of VKA therapy in VTE and CKD, although all NOACs are renal excreted to some extent, which introduces the risk of accumulation and relative overdosing. Pre-clinical data indicate that the rate of renal excretion of active NOAC varies from 27\% (apixaban) over 35\% (rivaroxaban) and 50\% (edoxaban) to $80 \%$ (dabigatran). ${ }^{63,76-78}$ While the high rate of renal excretion for dabigatran has clinical implications, the considerably lower renal excretion rates seen in direct Xa inhibitor (DXI) treatments carry a lower accumulation risk.

These pharmacological observations seem to translate into a profound clinical benefit from VTE treatment with DXI in CKD.

Rivaroxaban: In a dedicated post hoc analysis of the pooled EINSTEIN DVT/PE studies, ${ }^{79,80}$ increasing rates of recurrent VTE were observed in $\mathrm{CrCl}$ cohorts of $>80 ; 50$ to $79 ; 30$ to $49 ;<30 \mathrm{~mL} / \mathrm{min}$, which was observed in both rivaroxaban and VKA recipients to a similar degree. In contrast, with declining renal function rates of major bleeding dramatically increased from $1.0 \%$ (glomerular filtration rate $[\mathrm{GFR}]>80$ ) to $3.4 \%$ (GFR $<50 \mathrm{~mL} / \mathrm{min}$ ) in VKA recipients, but not in rivaroxaban-treated patients $(0.8 \%$ with GFR $>80$ compared with $0.9 \%$ with GFR $<50 \mathrm{~mL} / \mathrm{min}$ ).

Of note, impaired renal function was not a pre-defined criterion for rivaroxaban dose reduction so that these efficacy/safety findings were derived from standard dosing of rivaroxaban $15 \mathrm{mg}$ twice a day, followed by $20 \mathrm{mg}$ once a day throughout the study. As a consequence of this, the dosing label in the summary of product characteristics (SmPc) differs between VTE (no dose reduction in $\mathrm{CrCl}$ below $50 \mathrm{~mL} / \mathrm{min}$ in the acute phase, but dose reduction to $15 \mathrm{mg}$ once a day in the maintenance phase in $\mathrm{CrCl}$ below $30 \mathrm{~mL} /$ min is recommended for patients perceived to be at increased risk for bleeding complications) and atrial fibrillation treatment (dose reduction of rivaroxaban to $15 \mathrm{mg}$ for $\mathrm{CrCl}$ below $50 \mathrm{~mL} / \mathrm{min}$ ). Rivaroxaban is not recommended for patients with a $\mathrm{CrCl}$ of $<15 \mathrm{~mL} / \mathrm{min}$ in both indications.

Apixaban: In its phase III trial, apixaban was found to have similar efficacy and better safety compared with LMWH/VKA in patients treated for DVT and PE. ${ }^{68}$ Although no dedicated post hoc analysis exist for CKD patients, the appendix of this publication also includes sub-group analyses on patients with renal impairment. Overall, apixaban efficacy was established across the evaluated spectrum of renal function $(\mathrm{CrCl}$ $<30 \mathrm{~mL} / \mathrm{min}$ was an exclusion criterion) but a significant reduction of bleeding events by apixaban was only seen in patients with normal renal function, whereas bleeding rates similarly increased for apixaban and LMWH/VKA as renal function declined. ${ }^{68,81}$

A dose reduction of apixaban from $5 \mathrm{mg}$ twice a day to $2.5 \mathrm{mg}$ twice a day is recommended if two of the three following criteria are fulfilled: body weight $<60 \mathrm{~kg}$, age $>80$ years and/or creatinine level $>133 \mu \mathrm{mol} / \mathrm{L}$ or $>1.5 \mathrm{mg} / \mathrm{dL}$.

Edoxaban: The third DXI, edoxaban, also demonstrated noninferiority in the effectiveness of VTE therapy compared with LMWH/VKA with significantly reduced bleeding rates in edoxaban recipients. ${ }^{69}$ No dedicated post hoc analysis for CKD stages exist but in the appendix of the original trial report, a comparison of patients with $\mathrm{CrCl}$ higher or lower than $50 \mathrm{~mL} / \mathrm{min}$ is presented. Similar to the findings of the apixaban trial, edoxaban reached consistent efficacy with non-inferiority towards LMWH/VKA in both sub-groups, whereas a significant reduction in bleeding complications was observed only in patients with normal renal function. With declining renal function, bleeding rates numerically increased for edoxaban and LMWH/VKA. ${ }^{69}$ In a sub-group analysis, therapy with $30 \mathrm{mg}$ of edoxaban versus warfarin was compared for patients with a GFR 30 to $45 \mathrm{~mL} / \mathrm{min}$, a body weight $<60 \mathrm{~kg}$ or with accompanying therapy with P-glycoprotein inhibitors (pre-specified criteria for dose reduction of edoxaban; 733 vs. 719 patients). In the patient population for an edoxaban dose reduction, $30 \mathrm{mg}$ of edoxaban administration showed comparable efficacy with improved safety over warfarin. ${ }^{82}$ Based on these data, a dose 
reduction of edoxaban is recommended for patients with one of the following conditions: renal impairment with GFR 15 to $50 \mathrm{~mL} / \mathrm{min}$ or body weight $<60 \mathrm{~kg}$ or concomitant therapy with a P-gp inhibitor (ciclosporin, dronedarone, erythromycin, ketoconazole).

Of note, all DXI are recommended not be used in patients with a $\mathrm{CrCl}<15 \mathrm{~mL} / \mathrm{min}$ and should be used only with caution in patients with $\mathrm{CrCl} 15$ to $29 \mathrm{~mL} / \mathrm{min}$. Only few data exist for DXI treatment in patients under haemodialysis. ${ }^{83,84}$ Consequently, DXI should not be used in this population outside of dedicated registered clinical trials.

Dabigatran: Dabigatran is an orally available thrombin inhibitor. Two dedicated phase III studies in DVT and PE patients demonstrated non-inferiority of dabigatran versus LMWH/warfarin. In the pooled results of the RE-COVER 1 and 2 studies, a comparable effectiveness of dabigatran versus warfarin with improved safety profile was shown. ${ }^{85} \mathrm{Al}-$ though very limited information is available on the efficacy of dabigatran versus LMWH/VKA across different CKD stages, a sub-group analysis demonstrated a better safety profile of dabigatran in patients with a GFR $>50 \mathrm{~mL} / \mathrm{min}$ compared with VKA with similar safety in patients with a GFR 30 to $49 \mathrm{~mL} / \mathrm{min}$.

A dose reduction is recommended for patients who are older than 80 years or when co-administered with verapamil. Due to the higher renal elimination rate, dabigatran should not be used in patients with a $\mathrm{CrCl}$ below $30 \mathrm{~mL} / \mathrm{min}$.

\section{Conflict of Interest}

J.B.-W. has received honoraria and research support from Bayer, Boehringer Ingelheim, Daiichi Sankyo, LEO, Pfizer and Portola. S.W. has received honoraria and research support from Bayer, Daiichi Sankyo, LEO and Pfizer.

\section{References}

1 Kearon C, Akl EA, Ornelas J, et al. Antithrombotic therapy for VTE disease: Chest guideline and expert panel report. Chest 2016;149 (02):315-352

2 Konstantinides SV, Torbicki A, Agnelli G, et al; Task Force for the Diagnosis and Management of Acute Pulmonary Embolism of the European Society of Cardiology (ESC). 2014 ESC guidelines on the diagnosis and management of acute pulmonary embolism. Eur Heart J 2014;35(43):3033-3069, 3069a-3069k

3 Sullivan EA, Ford JB, Chambers G, Slaytor EK. Maternal mortality in Australia, 1973-1996. Aust N Z J Obstet Gynaecol 2004;44(05): 452-457, discussion 377

4 CEMACH. CEMACH Saving Mothers' Lives: Reviewing maternal deaths to make motherhood safer - 2003-2005. The Seventh Report of the Confidential Enquiries into Maternal Deaths in the United Kingdom London: Centre for Maternal and Child Enquiries; 2008. Available at: http://www.publichealth.hscni. net/sites/default/files/Saving\%20Mothers\%27\%20Lives\%20200305\%20.pdf accessed on Nov 7; 2017

5 Heit JA, Kobbervig CE, James AH, Petterson TM, Bailey KR, Melton LJ III. Trends in the incidence of venous thromboembolism during pregnancy or postpartum: a 30-year population-based study. Ann Intern Med 2005;143(10):697-706

6 Wells PS, Anderson DR, Rodger M, et al. Excluding pulmonary embolism at the bedside without diagnostic imaging: management of patients with suspected pulmonary embolism presenting to the emergency department by using a simple clinical model and D-dimer. Ann Intern Med 2001;135(02):98-107

7 Wells PS, Anderson DR, Rodger M, et al. Evaluation of D-dimer in the diagnosis of suspected deep-vein thrombosis. N Engl J Med 2003;349(13):1227-1235

8 Le Gal G, Righini M, Roy PM, et al. Prediction of pulmonary embolism in the emergency department: the revised Geneva score. Ann Intern Med 2006;144(03):165-171

9 Chabloz P, Reber G, Boehlen F, Hohlfeld P, de Moerloose P. TAFI antigen and D-dimer levels during normal pregnancy and at delivery. Br J Haematol 2001;115(01):150-152

10 Chan WS, Chunilal S, Lee A, Crowther M, Rodger M, Ginsberg JS. A red blood cell agglutination D-dimer test to exclude deep venous thrombosis in pregnancy. Ann Intern Med 2007;147(03):165-170

11 Chan WS, Lee A, Spencer FA, et al. D-dimer testing in pregnant patients: towards determining the next 'level' in the diagnosis of deep vein thrombosis. J Thromb Haemost 2010;8(05):1004-1011

12 Bates SM, Jaeschke R, Stevens SM, et al. Diagnosis of DVT: Antithrombotic Therapy and Prevention of Thrombosis, 9th ed: American College of Chest Physicians Evidence-Based Clinical Practice Guidelines. Chest 2012;141(2, Suppl):e351S-e418S

13 Linnemann B, Bauersachs R, Rott H, et al; Working Group in Women's Health of the Society of Thrombosis and Haemostasis. Diagnosis of pregnancy-associated venous thromboembolism - position paper of the Working Group in Women's Health of the Society of Thrombosis and Haemostasis (GTH). Vasa 2016;45(02):87-101

14 Einstein AJ, Henzlova MJ, Rajagopalan S. Estimating risk of cancer associated with radiation exposure from 64-slice computed tomography coronary angiography. JAMA 2007;298(03):317-323

15 Ginsberg JS, Hirsh J, Rainbow AJ, Coates G. Risks to the fetus of radiologic procedures used in the diagnosis of maternal venous thromboembolic disease. Thromb Haemost 1989;61(02):189-196

16 Kanal E, Barkovich AJ, Bell C, et al; ACR Blue Ribbon Panel on MR Safety. ACR guidance document for safe MR practices: 2007. AJR Am J Roentgenol 2007;188(06):1447-1474

17 Romualdi E, Dentali F, Rancan E, et al. Anticoagulant therapy for venous thromboembolism during pregnancy: a systematic review and a meta-analysis of the literature. J Thromb Haemost 2013;11(02):270-281

18 Bates SM, Greer IA, Middeldorp S, Veenstra DL, Prabulos AM, Vandvik PO. Recommendations for prophylaxis of pregnancyrelated venous thromboembolism in carriers of inherited thrombophilia. Comment on the 2012 ACCP guidelines: a rebuttal. J Thromb Haemost 2013;11(09):1782-1784

19 Astedt B. Thrombotic and hemostatic problems in pregnancy and labor and their significance for the fetus and newborn infant [in German]. Padiatr Padol 1990;25(01):43-53

20 Bates SM, Ginsberg JS. Anticoagulants in pregnancy: fetal effects. Baillieres Clin Obstet Gynaecol 1997;11(03):479-488

21 Pauli RM, Hall JG. Warfarin embryopathy. Lancet 1979;2 (8134): 144

22 Mehndiratta S, Suneja A, Gupta B, Bhatt S. Fetotoxicity of warfarin anticoagulation. Arch Gynecol Obstet 2010;282(03):335-337

23 Bapat P, Kedar R, Lubetsky A, et al. Transfer of dabigatran and dabigatran etexilate mesylate across the dually perfused human placenta. Obstet Gynecol 2014;123(06):1256-1261

24 Bapat P, Pinto LS, Lubetsky A, Berger H, Koren G. Rivaroxaban transfer across the dually perfused isolated human placental cotyledon. Am J Obstet Gynecol 2015;213(05):710.e1-710.e6

25 Bapat P, Pinto LS, Lubetsky A, et al. Examining the transplacental passage of apixaban using the dually perfused human placenta. J Thromb Haemost 2016;14(07):1436-1441

26 Bayer Pharma AG. Xarelto summary of product characteristics; 2016. Available at: http://www.medicines.org.uk/emc/medicine/ 25586/SPC

27 Bristol-Myers Squibb-Pfizer. Eliquis summary of product characteristics; 2016. Available at: http://www.medicines.org.uk/emc/ medicine/24988 
28 Boehringer-Ingelheim. Pradaxa summary of product characteristics; 2016. Available at: http://www.medicines.org.uk/EMC/ medicine/24839/SPC/Pradaxa +150

29 Beyer-Westendorf J, Michalski F, Tittl L, et al. Pregnancy outcome in patients exposed to direct oral anticoagulants - and the challenge of event reporting. Thromb Haemost 2016;116(04): 651-658

30 Cohen H, Arachchillage DR, Beyer-Westendorf J, Middeldorp S, Kadir RA. Direct oral anticoagulants and women. Semin Thromb Hemost 2016;42(07):789-797

31 Khorana AA, Francis CW, Culakova E, Kuderer NM, Lyman GH. Thromboembolism is a leading cause of death in cancer patients receiving outpatient chemotherapy. J Thromb Haemost 2007;5 (03):632-634

32 Timp JF, Braekkan SK, Versteeg HH, Cannegieter SC. Epidemiology of cancer-associated venous thrombosis. Blood 2013;122(10): 1712-1723

33 Prandoni P, Lensing AW, Piccioli A, et al. Recurrent venous thromboembolism and bleeding complications during anticoagulant treatment in patients with cancer and venous thrombosis. Blood 2002;100(10):3484-3488

34 Lyman GH, Eckert L, Wang Y, Wang H, Cohen A. Venous thromboembolism risk in patients with cancer receiving chemotherapy: a real-world analysis. Oncologist 2013;18(12):1321-1329

35 Cronin-Fenton DP, Søndergaard F, Pedersen LA, et al. Hospitalisation for venous thromboembolism in cancer patients and the general population: a population-based cohort study in Denmark, 1997-2006. Br J Cancer 2010;103(07):947-953

36 Chew HK, Wun T, Harvey D, Zhou H, White RH. Incidence of venous thromboembolism and its effect on survival among patients with common cancers. Arch Intern Med 2006;166(04):458-464

37 Louzada ML, Majeed H, Dao V, Wells PS. Risk of recurrent venous thromboembolism according to malignancy characteristics in patients with cancer-associated thrombosis: a systematic review of observational and intervention studies. Blood Coagul Fibrinolysis 2011;22(02):86-91

38 Douma RA, van Sluis GL, Kamphuisen PW, et al. Clinical decision rule and D-dimer have lower clinical utility to exclude pulmonary embolism in cancer patients. Explanations and potential ameliorations. Thromb Haemost 2010;104(04):831-836

39 Wilts IT, Le Gal G, Den Exter PL, et al. Performance of the ageadjusted cut-off for D-dimer in patients with cancer and suspected pulmonary embolism. Thromb Res 2017;152:49-51

40 Heit JA. Predicting the risk of venous thromboembolism recurrence. Am J Hematol 2012;87(Suppl 1):S63-S67

41 Heit JA, Lahr BD, Petterson TM, Bailey KR, Ashrani AA, Melton LJ III. Heparin and warfarin anticoagulation intensity as predictors of recurrence after deep vein thrombosis or pulmonary embolism: a population-based cohort study. Blood 2011;118(18):4992-4999

42 Gussoni G, Frasson S, La Regina M, Di Micco P, Monreal M; RIETE Investigators. Three-month mortality rate and clinical predictors in patients with venous thromboembolism and cancer. Findings from the RIETE registry. Thromb Res 2013;131(01):24-30

43 Trujillo-Santos J, Nieto JA, Tiberio G, et al; RIETE Registry. Predicting recurrences or major bleeding in cancer patients with venous thromboembolism. Findings from the RIETE Registry. Thromb Haemost 2008;100(03):435-439

44 Aujesky D, Obrosky DS, Stone RA, et al. Derivation and validation of a prognostic model for pulmonary embolism. Am J Respir Crit Care Med 2005;172(08):1041-1046

45 Jiménez D, Aujesky D, Moores L, et al; RIETE Investigators. Simplification of the pulmonary embolism severity index for prognostication in patients with acute symptomatic pulmonary embolism. Arch Intern Med 2010;170(15):1383-1389

46 Carrier M, Righini M, Wells PS, et al. Subsegmental pulmonary embolism diagnosed by computed tomography: incidence and clinical implications. A systematic review and meta-analysis of the management outcome studies. J Thromb Haemost 2010;8 (08):1716-1722

47 den Exter PL, Hooijer J, Dekkers OM, Huisman MV. Risk of recurrent venous thromboembolism and mortality in patients with cancer incidentally diagnosed with pulmonary embolism: a comparison with symptomatic patients. J Clin Oncol 2011;29(17): 2405-2409

48 Cronin CG, Lohan DG, Keane M, Roche C, Murphy JM. Prevalence and significance of asymptomatic venous thromboembolic disease found on oncologic staging CT. AJR Am J Roentgenol 2007; 189(01):162-170

49 Douma RA, Kok MG, Verberne LM, Kamphuisen PW, Büller HR. Incidental venous thromboembolism in cancer patients: prevalence and consequence. Thromb Res 2010;125(06):e306-e309

50 Di Nisio M, Ferrante N, De Tursi M, et al. Incidental venous thromboembolism in ambulatory cancer patients receiving chemotherapy. Thromb Haemost 2010;104(05):1049-1054

51 Browne AM, Cronin CG, English C, NiMhuircheartaigh J, Murphy JM, Bruzzi JF. Unsuspected pulmonary emboli in oncology patients undergoing routine computed tomography imaging. J Thorac Oncol 2010;5(06):798-803

52 Dentali F, Ageno W, Giorgi Pierfranceschi M, et al. Prognostic relevance of an asymptomatic venous thromboembolism in patients with cancer. J Thromb Haemost 2011;9(05):1081-1083

53 Callejas MF, Errázuriz JI, Castillo F, et al. Incidental venous thromboembolism detected by PET-CT in patients with cancer: prevalence and impact on survival rate. Thromb Res 2014;133 (05):750-755

54 Lyman GH, Khorana AA, Kuderer NM, et al; American Society of Clinical Oncology Clinical Practice. Venous thromboembolism prophylaxis and treatment in patients with cancer: American Society of Clinical Oncology clinical practice guideline update. J Clin Oncol 2013;31(17):2189-2204

55 Lee AY, Levine MN, Baker RI, et al; Randomized Comparison of Low-Molecular-Weight Heparin versus Oral Anticoagulant Therapy for the Prevention of Recurrent Venous Thromboembolism in Patients with Cancer (CLOT) Investigators. Low-molecular-weight heparin versus a coumarin for the prevention of recurrent venous thromboembolism in patients with cancer. N Engl J Med 2003;349 (02):146-153

56 Lee AY, Bauersachs R, Janas MS, et al; CATCH Investigators. CATCH: a randomised clinical trial comparing long-term tinzaparin versus warfarin for treatment of acute venous thromboembolism in cancer patients. BMC Cancer 2013;13:284

57 Posch F, Königsbrügge O, Zielinski C, Pabinger I, Ay C. Treatment of venous thromboembolism in patients with cancer: a network meta-analysis comparing efficacy and safety of anticoagulants. Thromb Res 2015;136(03):582-589

58 Bott-Kitslaar DM, Saadiq RA, McBane RD, et al. Efficacy and safety of rivaroxaban in patients with venous thromboembolism and active malignancy: a single-center registry. Am J Med 2016;129 (06):615-619

59 Mahé I, Chidiac J, Bertoletti L, et al; RIETE Investigators. The clinical course of venous thromboembolism may differ according to cancer site. Am J Med 2017;130(03):337-347

60 Mantha S, Laube E, Miao Y, et al. Safe and effective use of rivaroxaban for treatment of cancer-associated venous thromboembolic disease: a prospective cohort study. J Thromb Thrombolysis 2017;43(02):166-171

61 Raskob GE, van Es N, Verhamme P, et al. Edoxaban for the treatment of cancer-associated venous thromboembolism. N Engl J Med 2018; 378:615-624

62 Young A, Marshall A, Thirlwall J, et al. Anticoagulation therapy in selected cancer patients at risk of recurrence of venous thromboembolism: results of the Select- $\mathrm{D}^{\mathrm{TM}}$ pilot trial. Annual Meeting of the American Society of Hematology; 2017; oral abstract no. 625 
63 Heidbuchel H, Verhamme P, Alings M, et al. Updated European Heart Rhythm Association Practical Guide on the use of nonvitamin $\mathrm{K}$ antagonist anticoagulants in patients with non-valvular atrial fibrillation. Europace 2015;17(10):1467-1507

64 Lutz J, Menke J, Sollinger D, Schinzel H, Thürmel K. Haemostasis in chronic kidney disease. Nephrol Dial Transplant 2014;29(01):29-40

65 Monreal M, Falgá C, Valle R, et al; RIETE Investigators. Venous thromboembolism in patients with renal insufficiency: findings from the RIETE Registry. Am J Med 2006;119(12):1073-1079

66 Bauersachs R, Berkowitz SD, Brenner B, et al; EINSTEIN Investigators. Oral rivaroxaban for symptomatic venous thromboembolism. N Engl J Med 2010;363(26):2499-2510

67 Büller HR, Prins MH, Lensin AW, et al; EINSTEIN-PE Investigators. Oral rivaroxaban for the treatment of symptomatic pulmonary embolism. N Engl J Med 2012;366(14):1287-1297

68 Agnelli G, Buller HR, Cohen A, et al; AMPLIFY Investigators. Oral apixaban for the treatment of acute venous thromboembolism. N Engl J Med 2013;369(09):799-808

69 Büller HR, Décousus H, Grosso MA, et al; Hokusai-VTE Investigators. Edoxaban versus warfarin for the treatment of symptomatic venous thromboembolism. N Engl J Med 2013;369(15):1406-1415

70 Schulman S, Kakkar AK, Goldhaber SZ, et al; RE-COVER II Trial Investigators. Treatment of acute venous thromboembolism with dabigatran or warfarin and pooled analysis. Circulation 2014;129 (07):764-772

71 Schulman S, Kearon C, Kakkar AK, et al; RE-COVER Study Group. Dabigatran versus warfarin in the treatment of acute venous thromboembolism. N Engl J Med 2009;361(24):2342-2352

72 Chan KE, Lazarus JM, Thadhani R, Hakim RM. Warfarin use associates with increased risk for stroke in hemodialysis patients with atrial fibrillation. J Am Soc Nephrol 2009;20(10):2223-2233

73 Hijazi Z, Oldgren J, Lindbäck J, et al; ARISTOTLE and RE-LY Investigators. The novel biomarker-based ABC (age, biomarkers, clinical history)-bleeding risk score for patients with atrial fibrillation: a derivation and validation study. Lancet 2016;387(10035):2302-2311

74 El-Abbadi M, Giachelli CM. Mechanisms of vascular calcification. Adv Chronic Kidney Dis 2007;14(01):54-66

75 Brodsky SV, Nadasdy T, Rovin BH, et al. Warfarin-related nephropathy occurs in patients with and without chronic kidney disease and is associated with an increased mortality rate. Kidney Int 2011;80(02):181-189
76 Kubitza D, Becka M, Mueck W, et al. Effects of renal impairment on the pharmacokinetics, pharmacodynamics and safety of rivaroxaban, an oral, direct Factor Xa inhibitor. Br J Clin Pharmacol 2010; 70(05):703-712

77 Chang M, Yu Z, Shenker A, et al. Effect of renal impairment on the pharmacokinetics, pharmacodynamics, and safety of apixaban. J Clin Pharmacol 2016;56(05):637-645

78 Stangier J, Rathgen K, Stähle H, Mazur D. Influence of renal impairment on the pharmacokinetics and pharmacodynamics of oral dabigatran etexilate: an open-label, parallel-group, single-centre study. Clin Pharmacokinet 2010;49(04):259-268

79 Prins MH, Lensing AW, Bauersachs R, et al; EINSTEIN Investigators. Oral rivaroxaban versus standard therapy for the treatment of symptomatic venous thromboembolism: a pooled analysis of the EINSTEIN-DVT and PE randomized studies. Thromb J 2013;11 (01):21

80 Bauersachs RM, Lensing AW, Prins MH, et al. Rivaroxaban versus enoxaparin/vitamin $\mathrm{K}$ antagonist therapy in patients with venous thromboembolism and renal impairment. Thromb J 2014;12:25

81 Pathak R, Pandit A, Karmacharya P, et al. Meta-analysis on risk of bleeding with apixaban in patients with renal impairment. Am J Cardiol 2015;115(03):323-327

82 Verhamme P, Wells PS, Segers A, et al. Dose reduction of edoxaban preserves efficacy and safety for the treatment of venous thromboembolism. An analysis of the randomised, double-blind HOKUSAI VTE trial. Thromb Haemost 2016;116(04):747-753

83 De Vriese AS, Caluwé R, Bailleul E, et al. Dose-finding study of rivaroxaban in hemodialysis patients. Am J Kidney Dis 2015;66 (01):91-98

84 Wang X, Tirucherai G, Marbury TC, et al. Pharmacokinetics, pharmacodynamics, and safety of apixaban in subjects with end-stage renal disease on hemodialysis. J Clin Pharmacol 2016;56(05):628-636

85 Goldhaber SZ, Schellong S, Kakkar A, et al. Treatment of acute pulmonary embolism with dabigatran versus warfarin. A pooled analysis of data from RE-COVER and RE-COVER II. Thromb Haemost 2016;116(04):714-721

86 Henriksson P, Westerlund E, Wallén H, Brandt L, Hovatta O, Ekbom A. Incidence of pulmonary and venous thromboembolism in pregnancies after in vitro fertilisation: cross sectional study. BMJ 2013 Jan 15;346(04):e8632. doi: 10.1136/bmj.e8632 